adults allowing us to better understand the brains of over half of the world's population that speaks more than one language. OBJECTIVES/GOALS: Literature is mixed regarding a bilingual advantage in executive control (EC). While it has been shown that young adult bilinguals have greater gray matter volume (GMV) than monolinguals in EC regions, there is behavioral evidence that suggests such difference would be more pronounced in children. METHODS/STUDY POPULATION: Using SPM12 to test this hypothesis, we used a whole-brain t-test to compare GMV in 35 English-speaking monolingual and 20 Spanish-English early (learned both languages before 6 years old) bilingual children. Next, we submitted both groups of children to an ANOVA with 42 English speaking monolingual and 26 Spanish-English bilingual adults to test for an interaction of Language Experience by Age Group at the level of the whole brain. RESULTS/ANTICIPATED RESULTS: e between-group comparison of bilingual and monolingual children, revealed more GMV in bilingual compared to monolingual children in regions associated with EC (right middle and inferior frontal gyri, superior parietal lobule, and precuneus). Our second analysis, an ANOVA comparing bilingual and monolingual children and adults, revealed an interaction in which bilingual $>$ monolingual GMV in children was greater than any bilingual $>$ monolingual GMV (or bilingual=monolingual GMV) in the adult groups in the right superior parietal lobule (BA1). No regions indicated that bilingual $>$ monolingual GMV was more pronounced in adults. DISCUSSION/SIGNIFICANCE OF FINDINGS: These results provide further evidence for GMV differences in early bilinguals in regions associated with EC and indicate that more GMV differences exist between bilingual and monolingual children than adults.

16886

\section{Moyamoya-Like Vasculopathies Observed In a Novel Mouse Surgical Model}

Justin F. Fraser, Laura Whitnel and *Jill Roberts

University of Kentucky

ABSTRACT IMPACT: Development of our animal model of moyamoya will provide a meaningful assessment of therapeutic efficacy of interventions applicable to the clinical setting. OBJECTIVES/ GOALS: Moyamoya is a cerebrovascular condition with progressive stenosis of the internal carotid arteries (ICA) and formation of abnormal vascular collaterals at the base of the brain, all of which result in ischemic and hemorrhagic strokes. We aim to develop a needed animal model of this condition in order to develop new therapeutics. METHODS/STUDY POPULATION: Male and female C57Bl/6J mice (4 months old) underwent surgery for the unilateral placement of a microcoil $(0.16 \mathrm{~mm}$ ID) onto the proximal ICA or sham control. After 30 and 60 days $(\mathrm{N}=6-8 /$ time point), the brain blood vessels were examined for changes in diameter, number of anastomoses, and development of new collaterals using DiI stain. Brain tissue was examined for micro-hemorrhages using Prussian blue stain, and cross-sections of blood vessels were examined for intimal thickening using $\mathrm{H} \& \mathrm{E}$ and smooth muscle actin. Expression of vascular endothelial growth factor (VEGF), which is associated with angiogenesis and moyamoya syndrome, was quantified by qPCR. Blood samples were also analyzed for inflammatory biomarkers using ELISA. RESULTS/ANTICIPATED RESULTS: Within 30 days, the distal ICA and anterior cerebral artery (ACA) had significantly decreased diameters at the Circle of Willis, with an initial decrease in the number of cortical anastomoses. Histology demonstrated smaller lumen diameter and alterations to in the various layers of the blood vessels, indicating intimal thickening and stenosis of the affected blood vessels. There was also a significant increase in the number of intracranial micro-bleeds, suggesting a compromised vascular integrity. This may be due, in part, to a significant upregulation in VEGF gene expression within the striatum, a region of hemorrhagic occurrence in moyamoya patients. DISCUSSION/SIGNIFICANCE OF FINDINGS: We report the development of an animal model with vasculopathies that mimic those observed in patients with moyamoya syndrome. With further characterization, this animal model will have a positive impact as a meaningful assessment of therapeutic efficacy of interventions applicable to the clinical setting.

38227

Specific and highly potent human monoclonal antibodies against SARS-CoV-2

Mayara Garcia de Mattos Barbosa ${ }^{1}$, Hui Liu, Daniel Huynh ${ }^{1}$, Jeffrey L. Platt ${ }^{2}$ and Marilia Cascalho ${ }^{2}$

${ }^{1}$ Department of Surgery, University of Michigan and ${ }^{2}$ Departments of Surgery and Microbiology and Immunology, University of Michigan

ABSTRACT IMPACT: We devised a new method to produce highly potent SARS-CoV2-specific that can be used to treat severely ill patients with Covid-19. OBJECTIVES/GOALS: Neutralizing antibodies against SARS-CoV-2 are thought to offer the most immediate and effective treatment for those severely afflicted by Covid-19. We devised an approach for rapid and efficient generation of human monoclonal antibodies with neutralizing activity against SARS-CoV-2. METHODS/STUDY POPULATION: SARS-CoV-2 S1 spike protein-specific memory B cells were isolated from 12 subjects recovering from infection with that virus. Paired end single index sequencing was performed using up to 10,000 antigen-specific $B$ cells per subject. Antigen-specific B cell clones were identified by unique diversity and joining gene $\mathrm{V}(\mathrm{D}) \mathrm{J}$ rearrangements and the CDR3 regions. $\mathrm{VH}$ and VL regions were cloned and the products expressed in 293T/17 cells to generate spike-specific human monoclonal antibodies. RESULTS/ANTICIPATED RESULTS: Fortythree human monoclonal antibodies were produced. Every monoclonal antibody so generated neutralized viruses pseudotyped with Spike protein of the Wuhan-1 strain. Eighteen monoclonal antibodies neutralized pseudotyped viruses with half-maximal inhibitory concentration (IC50s) between $1 \mathrm{pg} / \mathrm{mL}$ and $1 \mathrm{ng} / \mathrm{mL}(6.7 \mathrm{x}$ $10 \mathrm{E}-15 \mathrm{M}$ to $6.7 \times 10 \mathrm{E}-12 \mathrm{M}$ ), exceeding by $10-100$-fold the potency of previously reported anti-SARS-CoV-2-neutralizing monoclonal antibodies. Eight monoclonal antibodies neutralized viruses pseudotyped with mutant spike proteins previously identified in clinical isolates, including receptor binding domain mutants and the C-terminal D614G mutant with IC50<6.7 x10E-12M. DISCUSSION/SIGNIFICANCE OF FINDINGS: We show that SARS-CoV-2 evokes high affinity B cell responses. Some B cells produce antibodies that are broadly neutralizing; others produce strainspecific antibodies. However, antigenic variants that would potentially escape control by immunity or vaccination were nonetheless identified. 